\section{VALIDATION OF THE DYNAMIC MODEL OF THE PLANAR ROBOTIC ARM WITH USING GRAVITY TEST}

MONIKA TROJANOVA, TOMAS CAKURDA

Technical University of Kosice, Faculty of Manufacturing Technologies with a Seat in Presov, Department of Industrial Engineering and Informatics, Presov, Slovakia

DOI : 10.17973/MMSJ.2020_12_2020069

monika.trojanova@tuke.sk

The paper describes the validation of a dynamic model of a planar robotic arm using gravity tests. The drive of the arm (fluid muscles from the manufacturer Festo) was not activated during gravity tests (for the test was used only gravitation of solid). The measured data were obtained under the conditions that the joint angle was from $20^{\circ}$ to $40^{\circ}$, the measurements were performed 10 times for each angle and at the same time independent for both links. The dynamics simulation was performed in the MATLAB ${ }^{\circledR}$ and Simulink environment, using the created simulation scheme, which presented the dynamics of the manipulator's arm with the inclusion of the friction component. The simulation results were compared with the measured data using two criteria (MAE - Mean Absolute Error and $\mathrm{F}_{\text {gof }}$ - Goodness of Fit).

KEYWORDS

Dynamics, Fluidic Muscle, Gravity Test, Planar Robotic Arm, Validation

\section{INTRODUCTION}

The ability to control the movement of manipulators is conditioned by many aspects, e.g. detailed knowledge about its properties, correct simulation of motion, or correct derivation of the control algorithm. For simulating the motion (or also the design of the control algorithm) it is important to take into account the dynamic model. It is dynamic modeling (deriving equations) that explicitly describes the relationship between two basic parameters in the system, namely between motion and force. Within dynamic modeling, there are two basic approaches to how a dynamic model of manipulator can be expressed, namely the Newton-Euler formulation or the EulerLagrange formulation. The Euler-Lagrange formulation approaches the system as a whole and describes it based on potential energy and kinetic energy. In contrast, the NewtonEuler formulation is more challenging because it describes each term separately. The choice of method depends on many factors, e.g. the number of degrees of freedom of the manipulator, the topology of the placement of individual links and joints in the manipulator, and others. More about these methods is given in publications [Siciliano 2009] or in [Spong 2020].

Many researchers describe the issue of dynamics model of manipulators at different stages of the solution (they take into account friction, they take into account the dynamics of the arms or of the actuator, etc.). However, in order to create a complex dynamic model that would describe the system as much as possible, it is necessary to proceed with the sequence. Research in the field of dynamic modeling can be more complex and lengthy depending on the system, so some researchers' works do not include final dynamic models taking into account all the components, but parts of the research that are presented in several works.

Very interesting look at the issue of dynamic model identification for industrial robots - specifically for the KUKA robot is provided in the article [Swevers 2007], where the aim was to predict the torque joint in individual axes, while the predicted data were compared with measured data. However, this article also includes a part of the article devoted to the description of the dynamic model.

The authors in the article [Pitel 2014] present a dynamic model of actuators - pneumatic artificial muscles (PAMs), which form the drive of the manipulator (1-DOF) described in the article. They justify the need for the model by saying that the characteristics of PAM are highly nonlinear. They used for modeling various types of models compiled in the Matlab/Simulink environment (simple geometric model, Modified Hill's model, and others). The simulation results were compared with experimentally obtained data.

[Hosovsky 2014] describes experimental validation of a dynamic model for a manipulator with 1-DOF actuated with one pair of PAMs, where the dynamics of the system is controlled by nonlinear differential equations. The same author in a later publication [Hosovsky 2016] describes together with the team dynamic characterization and simulation of the 2-DOF soft robot arm, which is actuated by pneumatic artificial muscles. The article includes knowledge about the research of the manipulator arm dynamics, but also the dynamics of the actuator. The results of the investigation are compared with the system (measured data). The main goal of the research described in the article was used to create a comprehensive model of system dynamics that could be for the control phase. Subsequently, a one year later, was published the authors' work [Al-Qahtani 2017], which describes, among other things, the dynamic model of a four-link robotic manipulator, the base of which can be placed on the ground. As a drive was used in this manipulator commonly used type of actuator electromotor.They used the Euler-Lagrange formulation to describe the model.

Similar to the publication [Hosovsky 2016], where part of the article is devoted to the description of the dynamics of the manipulator's arm and the other part is devoted to the description of the dynamics of the actuator, as well as in the article [Leborne 2018]. The article describes dynamic modeling and identification of the manipulator arm, which is intended for use in specific conditions (underwater). The described manipulator is specific in that it is a heterogeneous control (different behavior in 3 joints).

The research described in this article is divided into 6 parts. After the introduction, which includes, among other things, publications that describe the dynamics and characteristics of manipulators, the second part briefly describes the investigated experimental system - planar robotic arm with 2 DOF and with fluidic muscle, specifically its parts and its basic principle of operation. The third part is devoted to basic knowledge in the field of modeling the kinematic structures of planar arms with two rotating joints. The fourth part describes the process of measuring data (joint angle as a function of time) on an experimental manipulator, and at the same time graphically interprets the course of these measured data. The fifth part of the article is devoted to the results of validation itself, and thus: a description of the indicators of evaluation of simulation results ( $F_{\text {gof }}$ and MAE), as well as the presentation of results in graphical form and based on indicators. The last part of the article is a summary - conclusion. 


\section{EXPERIMENTAL SETUP}

The object of the research is an experimental system - a planar robotic arm with two degrees of freedom, shown in Figure 1. The manipulator was assembled at the authors' workplace for experiments that would simulate the conditions of its application in industry, while the actuator of the manipulator was chosen unconventional type of actuators - pneumatic artificial muscles (PAM). Specifically, it is a modification of PAM - fluid muscles from the manufacturer's FESTO.

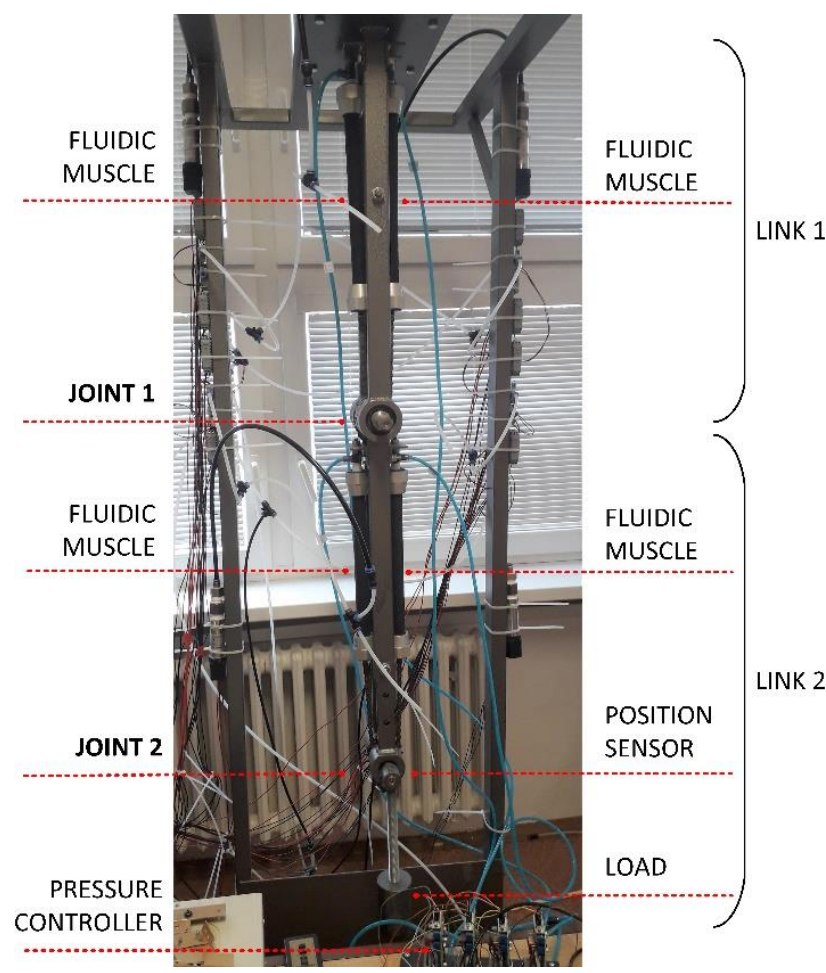

Figure 1. The experimental system - planar robotic arm with 2-DOF [Trojanova 2019]

The manipulator is divided into link 1 and link 2. The location of the manipulator was chosen so that it could be placed on the ceiling, and thus the upper arm of the manipulator is connected to the upper base. A weight (load) is attached at the end of the lower arm. The principle of operation of the manipulator is as follows: the compressor blows the working medium into two pairs of fluid muscles FESTO MAS-20; the contraction of the muscle creates a tensile force, which, however, is transmitted as torque due to the engagement of the joints, gears, and chains; the resulting joint angle is sensed by an incremental encoder; sensing and regulating the pressure in the muscles are provided by pressure regulators. [Trojanova 2018]

\section{DYNAMIC MATHEMATICAL MODEL OF THE SYSTEM}

The system described in the previous section represents a planar arm with two rotating joints, which is the most basic kinematic structure of manipulators. Figure 2 is a diagram of such a kinematic structure. To describe the dynamics of the manipulator's arm using a mathematical model, it is necessary to define the most basic parameters of the kinematic structure. An overview of the parameters used in the diagram, but also the mathematical formulation, is contained in Table 1.

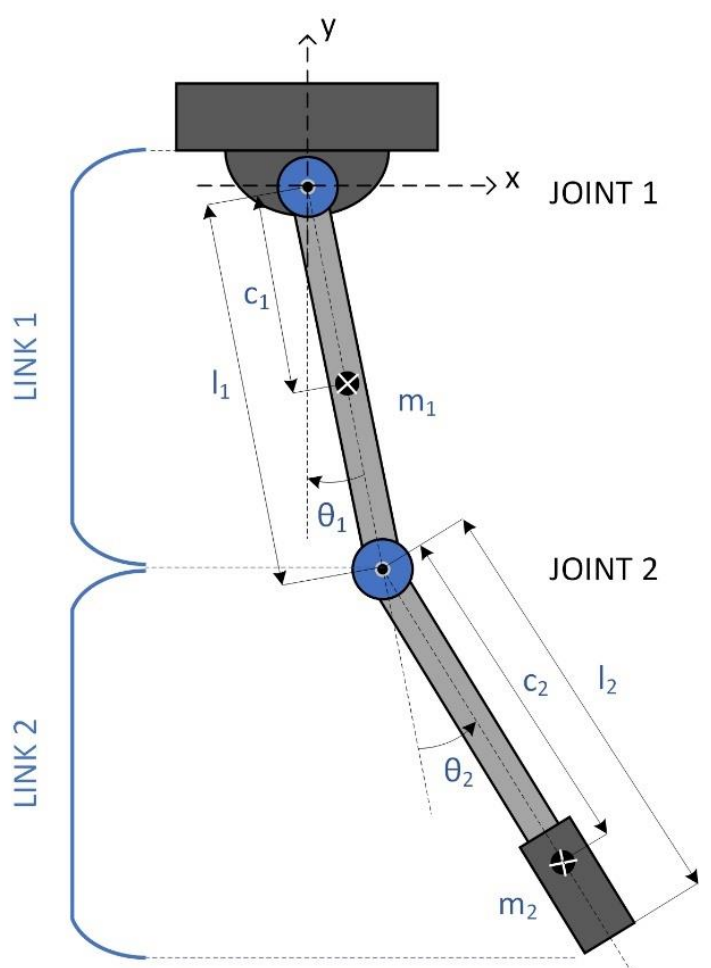

Figure 2. The scheme of planar robotic arm with 2-DOF

\begin{tabular}{|l|l|l|l|}
\hline $\boldsymbol{\theta}_{\mathbf{1}}$ & Joint 1 angle & $\boldsymbol{\theta}_{\mathbf{2}}$ & Joint 2 angle \\
\hline $\mathbf{m}_{\mathbf{1}}$ & Link 1 mass & $\mathbf{m}_{\mathbf{2}}$ & Link 2 mass \\
\hline $\mathbf{I}_{\mathbf{1}}$ & Link 1 length & $\mathbf{I}_{\mathbf{2}}$ & Link 2 length \\
\hline $\mathbf{c}_{\mathbf{1}}$ & Link 1 center of mass & $\mathbf{C}_{\mathbf{2}}$ & Link 2 center of mass \\
\hline $\boldsymbol{\tau}_{\mathbf{1}}$ & Joint 1 generalized force & $\mathbf{\tau}_{\mathbf{2}}$ & Joint 2 generalized force \\
\hline $\mathbf{I}_{\mathbf{1}}$ & $\begin{array}{l}\text { Link 1 moment of inertia } \\
\text { about axis z }\end{array}$ & $\mathbf{I}_{\mathbf{2}}$ & $\begin{array}{l}\text { Link 2 moment of inertia } \\
\text { about axis z }\end{array}$ \\
\hline $\mathbf{f c}_{\mathbf{1}}$ & $\begin{array}{l}\text { Viscous friction } \\
\text { coefficient in joint 1 }\end{array}$ & $\mathbf{f c}_{\mathbf{2}}$ & $\begin{array}{l}\text { Viscous friction } \\
\text { coefficient in joint 2 }\end{array}$ \\
\hline $\mathbf{f d}_{\mathbf{1}}$ & $\begin{array}{l}\text { Coulomb friction } \\
\text { magnitude in joint 1 }\end{array}$ & $\mathbf{f d}_{\mathbf{2}}$ & $\begin{array}{l}\text { Coulomb friction } \\
\text { magnitude in joint 2 }\end{array}$ \\
\hline $\mathbf{g}$ & Gravity constant & $\mathbf{F}$ & Friction term \\
\hline $\mathbf{G}$ & Gravity term & $\mathbf{\tau}$ & Generalized force term \\
\hline
\end{tabular}

Table 1. Overview of parameters listed in the dynamic model

To define a dynamic mathematical model of the kinematic structure of a planar arm with two rotating joints, the Lagrangian dynamic model presented in the literature [Kelly 2005] was used, where Equation 1 represents a general formulation of arm dynamics. The equations of motion for the link 1 and link 2 in the extended form are given in equation 2 (joint 1 generalized force $\tau_{1}$ ) and in equation 3 (joint 2 generalized force $\tau_{2}$ ).

$$
\begin{aligned}
M(\theta) & \ddot{\theta}+C(\theta, \dot{\theta}) \dot{\theta}+G(\theta)=\tau \\
\tau_{1}= & {\left[m_{1} c_{1}{ }^{2}+m_{2} l_{1}^{2}+m_{2} c_{2}{ }^{2}\right] \ddot{\theta}_{1} } \\
& +\left[2 m_{2} l_{1} c_{2} \cos \theta_{2}+I_{1}+I_{2}\right] \ddot{\theta}_{1} \\
& +\left[m_{2} c_{2}{ }^{2}+m_{2} l_{1} c_{2} \cos \theta_{2}+I_{2}\right] \ddot{\theta}_{2} \\
& -2 m_{2} l_{1} c_{2} \sin \theta_{2} \dot{\theta_{1}} \dot{\theta_{2}}-m_{2} l_{1} c_{2} \sin \theta_{2} \dot{\theta}_{2}^{2} \\
& +\left[m_{1} c_{1}+m_{2} l_{1}\right] g \sin \theta_{1}+m_{2} g c_{2} \sin \left(\theta_{1}+\theta_{2}\right) \\
\tau_{2}= & {\left[m_{2} c_{2}{ }^{2}+m_{2} l_{1} c_{2} \cos \theta_{2}+I_{2}\right] \ddot{\theta}_{1} } \\
& +\left[m_{2} c_{2}{ }^{2}+I_{2}\right] \ddot{\theta}_{2}+m_{2} l_{1} c_{2} \sin \theta_{2} \dot{\theta}_{1}^{2} \\
& +m_{2} g c_{2} \sin \left(\theta_{1}+\theta_{2}\right)
\end{aligned}
$$


The parameter $\tau$ in general (respectively $\tau_{1}$ and $\tau_{2}$ ) does not directly describe the torque of the drive, because it does not involve friction in the joints. These are just terms of force. However, the simulations were performed with a friction term $\mathrm{F}$, so equation 1 needs to be supplemented. The resulting shape of the dynamic mathematical model of the described arm is therefore equation 4 . Since the muscles were not activated during data measurement, the dynamics of these actuators were not considered in the simulations, only the gravity of the solids was used.

$$
M(\theta) \ddot{\theta}+C(\theta, \dot{\theta}) \dot{\theta}+G(\theta)+F(\dot{\theta})=\tau
$$

Friction term $\mathrm{F}$ is possible to detail describe by the next equation:

$$
F=\operatorname{sgn}(\omega) \cdot T_{c}+\beta \cdot \omega
$$

where:

$\omega$ represents the angular velocity in units $[\mathrm{rad} / \mathrm{s}]$,

- $\quad T_{c}$ is Coulomb friction magnitude in [N.m],

- $\quad B$ is Viscous friction coefficient in [N.m.s/rad].

The whole expression of Equation 5, that is on the right side of the equation, is known as Coulomb and viscous friction. [Papageorgiou 2020]

\section{MEASURED DATA}

The monitored output parameter for the manipulator was the joint angle. Using gravity tests, measurements were performed to obtain waveforms of this parameter independently for the link 1 and link 2 of the manipulator. During the tests, was initial joint angle in range for each of the link in the range of $20^{\circ}$ to $40^{\circ}$ in an interval of $10^{\circ}$. Within each joint angle, 10 measurements were performed, and the courses of these measurements were averaged.

The time at which the movement of the arm stabilized, and the measurement was completed (the joint angle was equal to $0^{\circ}$ ) was different, depending on the magnitude of the initial joint angle:

- The measurement time for a $20^{\circ}$ initial joint angle for both links was $\mathrm{T} 1=16.315 \mathrm{~s}$.

- The measurement time for a $30^{\circ}$ initial joint angle for both links was $\mathrm{T} 2=23.045 \mathrm{~s}$.

- The measurement time for a $40^{\circ}$ initial joint angle for both links was T3 $=26.995 \mathrm{~s}$.

The time dependences of the joint angle (from measurement) are presented by graphical dependencies. Each graph contains the waveforms of 10 measurements for a given initial joint angle (interpreted by curves of different colors) and one waveform of the arithmetic average of these 10 measurements (interpreted by a black curve marked as Average data).

For the link 1 were measured following waveforms:

- Figure 3 - at an initial joint angle of $20^{\circ}$,

- Figure 4 - at an initial joint angle of $30^{\circ}$,

- $\quad$ Figure 5 - at an initial joint angle of $40^{\circ}$.

For the link 2 were measured following waveforms:

\footnotetext{
Figure 6 - at an initial joint angle of $20^{\circ}$,
}

Figure 7 - at an initial joint angle of $30^{\circ}$,

- $\quad$ Figure 8 - at an initial joint angle of $40^{\circ}$.

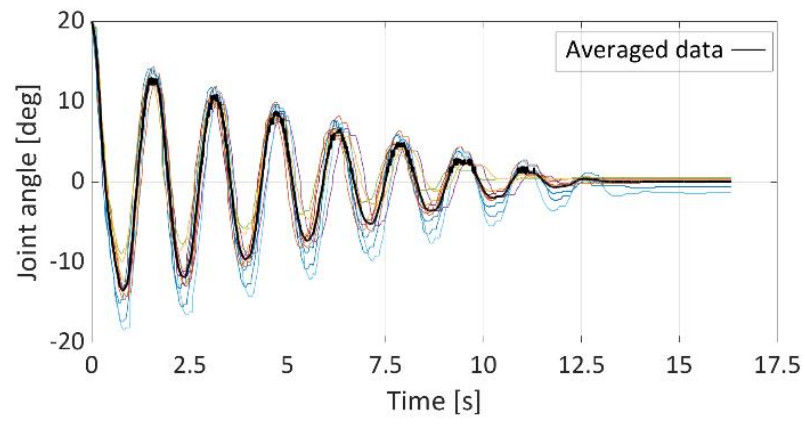

Figure 3. Measured values for link 1 and for the joint angle of $20^{\circ}$

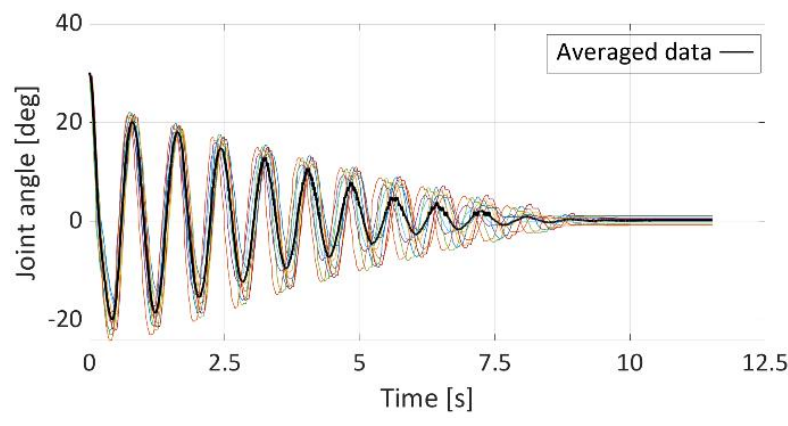

Figure 4. Measured values for link 1 and for the joint angle of $30^{\circ}$

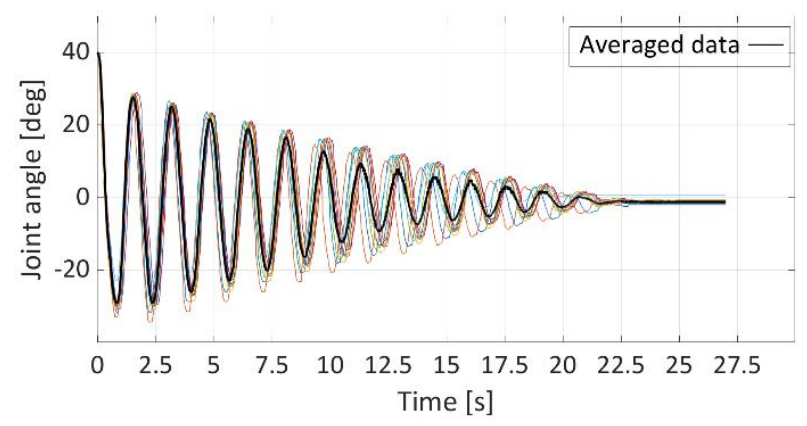

Figure 5. Measured values for link 1 and for the joint angle of $40^{\circ}$

Gravity tests within the link 1 showed that the joint angle had a more even course over time with gradual stabilization at $0^{\circ}$, as evidenced by the waveforms in Figure 3 - Figure 5. However, in the link 2, it can be seen that converge to steady-state begins only after overcoming the initial amplitude. (Figure 6 - Figure 8).

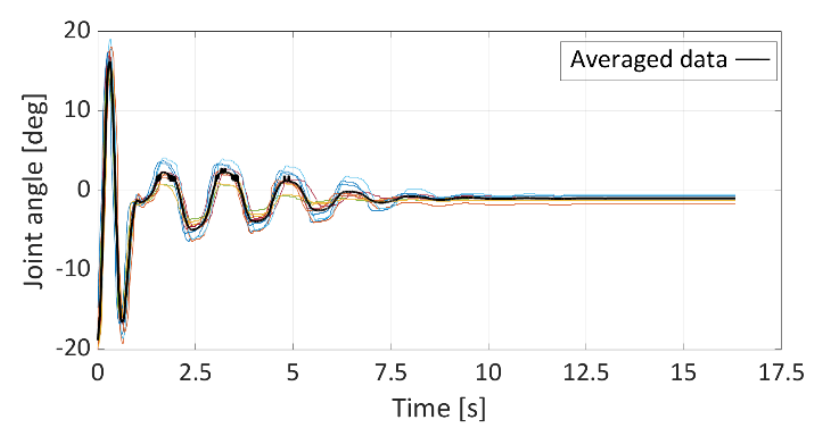

Figure 6. Measured values for link 2 and for the joint angle of $20^{\circ}$ 


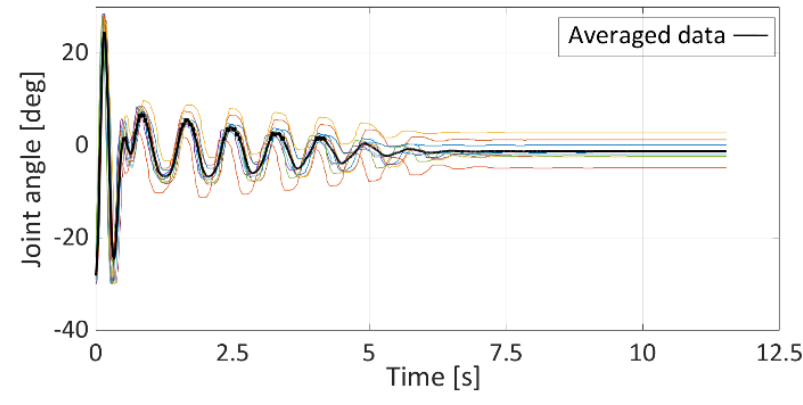

Figure 7. Measured values for link 2 and for the joint angle of $30^{\circ}$

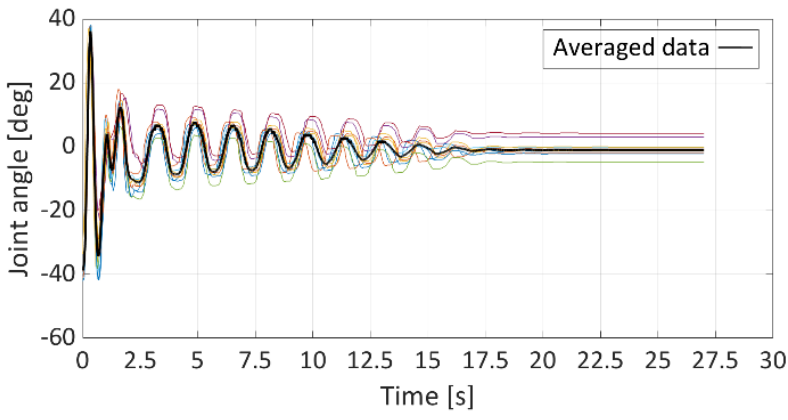

Figure 8. Measured values for link 2 and for the joint angle of $40^{\circ}$

\section{RESULTS OF VALIDATION FROM SIMULINK}

The measured data were processed in the Matlab environment and subsequently, a simulation scheme in the Simulink environment was created for the validation process based on a mathematical model (listed in the section Dynamic mathematical model of the system). Geometric input parameters were obtained from the CAD environment, where a $3 \mathrm{D}$ model of the system was made, the remaining parameters were determined experimentally. An overview of these input parameters with a designation, parameter size, and unit used is given in Table 2. The designation of the parameters was given in Table 1. A total of six simulations were performed:

- two simulations for the link 1 and link 2 (separately), for initial joint angle of $20^{\circ}$, when the simulation time was $\mathrm{T} 1=16.315 \mathrm{~s}$;

- two simulations for the link 1 and link 2 (separately), for initial joint angle of $30^{\circ}$, when the simulation time was $\mathrm{T} 2=23.045 \mathrm{~s}$;

- two simulations for the link 1 and link 2 (separately), for initial joint angle of $40^{\circ}$, when the simulation time was $\mathrm{T} 3=26.995 \mathrm{~s}$.

\begin{tabular}{|c|c|c|}
\hline Designation & Value & Unit \\
\hline $\mathbf{I}_{\mathbf{1}}$ & 0.240840 & $\mathrm{~kg} \cdot \mathrm{m}^{2}$ \\
\hline $\mathbf{I}_{\mathbf{2}}$ & 0.056052 & $\mathrm{~kg} \cdot \mathrm{m}^{2}$ \\
\hline $\mathbf{I}_{\mathbf{1}}$ & 0.374094 & $\mathrm{~m}$ \\
\hline $\mathbf{I c}_{\mathbf{2}}$ & 0.193949 & $\mathrm{~m}$ \\
\hline $\mathbf{I}_{\mathbf{1}}$ & 0.538534 & $\mathrm{~m}$ \\
\hline $\mathbf{f c}_{\mathbf{1}}$ & 0.725102 & $\mathrm{~N} \cdot \mathrm{m} \cdot \mathrm{s} \cdot \mathrm{rad}^{-1}$ \\
\hline $\mathbf{f d}_{\mathbf{1}}$ & 0.183334 & $\mathrm{~N} \cdot \mathrm{m}$ \\
\hline $\mathbf{f c}_{\mathbf{2}}$ & 0.235819 & $\mathrm{~N} \cdot \mathrm{m} \cdot \mathrm{s} \cdot \mathrm{rad}^{-1}$ \\
\hline $\mathbf{f d}_{\mathbf{2}}$ & 0.187307 & $\mathrm{~N} \cdot \mathrm{m}$ \\
\hline $\mathbf{m}_{\mathbf{1}}$ & 8.866883 & $\mathrm{~kg}$ \\
\hline $\mathbf{m}_{\mathbf{2}}$ & 6.743220 & $\mathrm{~kg}$ \\
\hline
\end{tabular}

Table 2. Overview of values of input parameters of simulink model
The validation outputs were exported from the Simulink to the Matlab environment in the form of matrices. Based on the exported data, were processed the graphical waveforms of simulated outputs, which were compared with the waveforms of data processed from the measured data. Figure 9 - Figure 14 represents the time dependencies of the joint angle (initial joint angle of $20^{\circ}, 30^{\circ}$, and $40^{\circ}$ ), where the blue curve represents the average data processed from the measurements, and the red curve represents the model output from the simulation.

Figure 9 - Figure 11 are the time dependencies of the joint angle for the link 1 at individual initial joint angles. It can be seen from the figures that the model can highly simulate the output based on the input parameters at all three examined joint angles compared to the average of the measured data (approx. about $70-80 \%)$. The model obtained the best result at joint angle of $30^{\circ}$ (Figure 10).

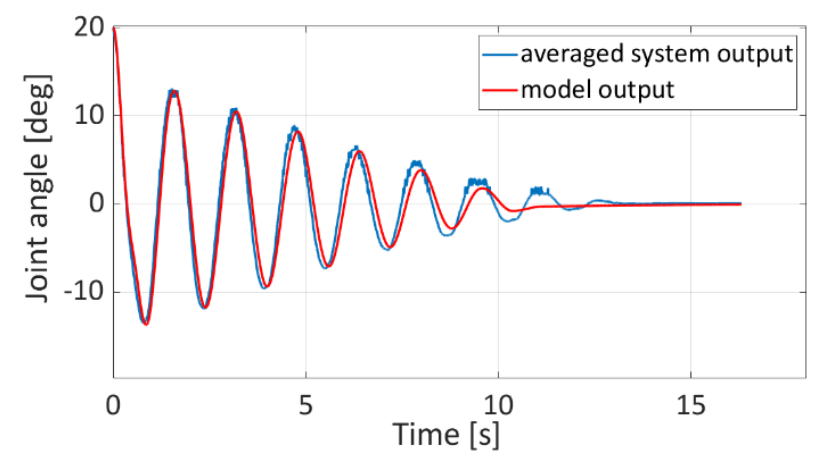

Figure 9. Time dependence of the joint angle of the link 1 for the averaged system output and for the output data (initial joint angle $20^{\circ}$ )

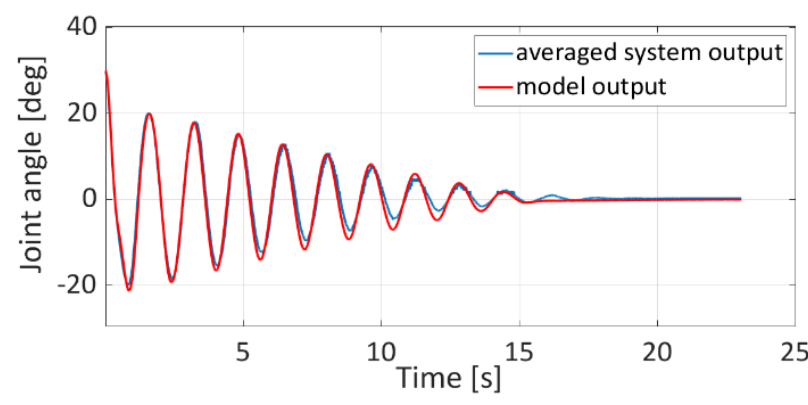

Figure 10. Time dependence of the joint angle of the link 1 for the averaged system output and for the output data (initial joint angle $30^{\circ}$ )

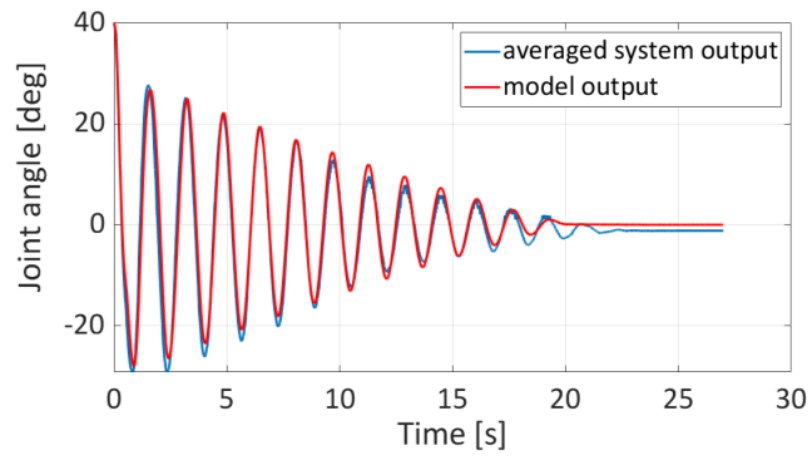

Figure 11. Time dependence of the joint angle of the link 1 for the averaged system output and for the output data (initial joint angle $40^{\circ}$ ) 
Figure 12 - Figure 14 represents the time dependencies of the output parameter joint angle for the link 2 at individual initial joint angles. Compared to the graphical interpretation of the link 1 , it can be seen that the output of the link 2 is not as good as it was with the link 1 (the reason may be oscillations as an oscillating component, which is more pronounced in the link 2). The agreement of the output of the link 2 in comparison with the average of the measured data is somewhere in the range of 50$70 \%$, while based on graphical interpretation, the best result is the output with a joint angle of $40^{\circ}$ angle (Figure 14).

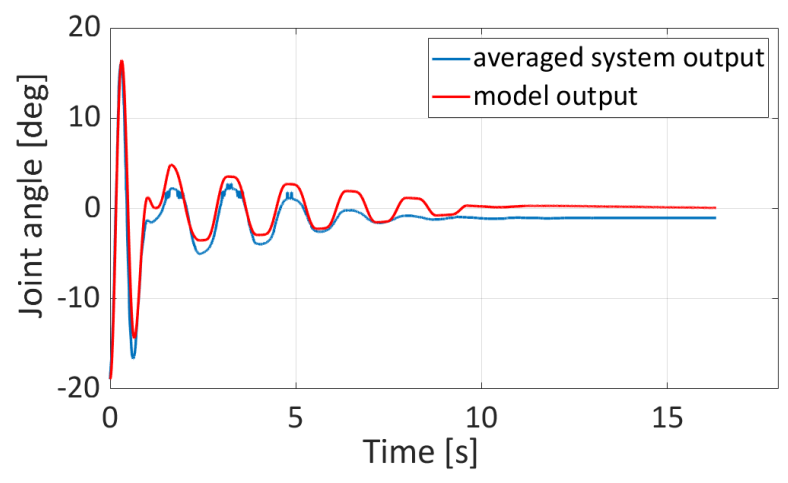

Figure 12. Time dependence of the joint angle of the link 2 for the averaged system output and for the output data (initial joint angle $20^{\circ}$ )

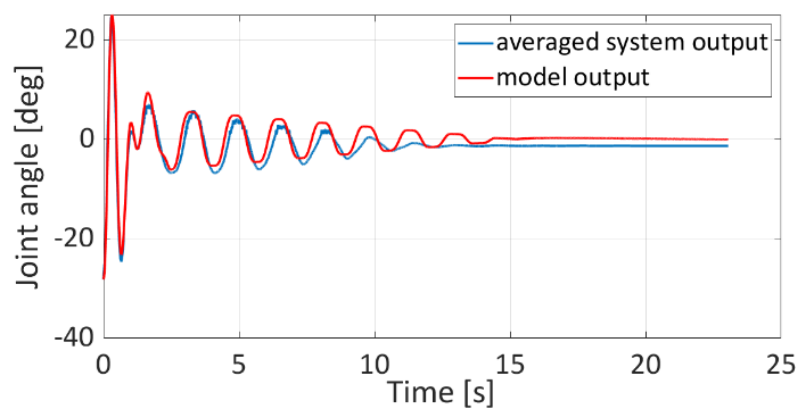

Figure 13. Time dependence of the joint angle of the link 2 for the averaged system output and for the output data (initial joint angle $30^{\circ}$ )

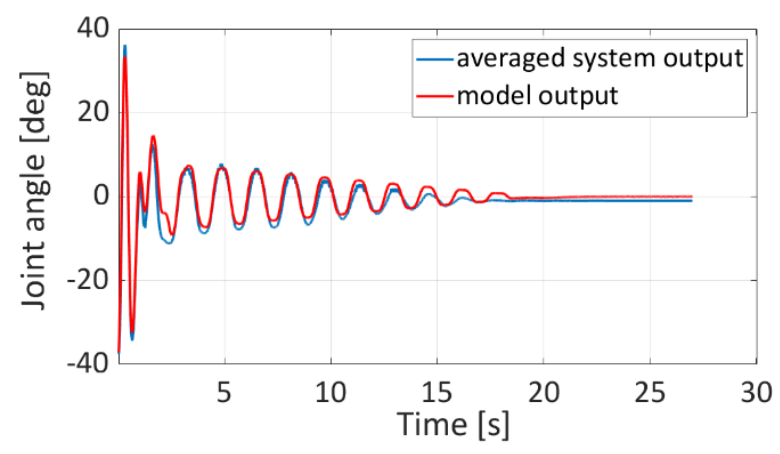

Figure 14. Time dependence of the joint angle of the link 1 for the averaged system output and for the output data (initial joint angle $40^{\circ}$ )

To compare the validation results, two criteria were chosen that compare the outputs of the system with the outputs of the model [Pal 2017]:

One of the criteria was the Goodness of Fit $\left(F_{\text {gof }}\right)$ indicator, expressed by equation 6 . This figure represents the Normal Root Mean Square Error (NRMSE) statistical indicator and compares the extent to which the model output is identical to the system output. The data has a percentage character, where $\mathrm{F}_{\text {gof }}=100 \%$ represents a complete agreement of the simulation and measurement results (desired state).
The second criterion chosen was the Mean Absolute Error (MAE) indicator, expressed by equation 7. The numerical value expresses the magnitude of the error that occurred as a result of the simulation of the output compared to the measured values. The smaller the value of the indicator, the smaller the error (the desired state is for $M A E=0$ ).

$$
\begin{aligned}
& F_{\text {gof }}=\left(1-\frac{\sqrt{\sum_{j=1}^{n}\left[y_{j}-\hat{y}_{j}\right]^{2}}}{\sqrt{\sum_{j=1}^{n}\left[y_{j}-\frac{1}{n} \sum_{j=1}^{n} y_{j}\right]^{2}}}\right) \times 100 \% \\
& M A E=\frac{1}{n} \sum_{j=1}^{n}\left|y_{j}-\hat{y}_{j}\right|
\end{aligned}
$$

Mathematical formulations of indicators contain the following parameters: $y_{j}$ system output on the $k$-th sample, $\hat{y}_{j}$ model output on the k-th sample, $\mathrm{n}$ - number of samples.

The results of the validation based on the $F_{\text {gof }}$ and MAE criteria are shown in Table 3 for the link 1 and Table 4 for the link 2. The output of the model achieved the best results for the link 1 based on the MAE criterion with an initial joint angle of $30^{\circ}$ when the value of $M A E=1.0049$. Based on the evaluation using the $F_{\text {gof }}$ criterion, the model output for the link 1 achieved the best result when the joint angle was equal to $30^{\circ}$ (agreement with the MAE result), and thus $\mathrm{F}_{\text {gof }}=81.28 \%$ (Table 3 ). For the link 2, the model achieved the best result (Table 4 ) at a joint angle of $20^{\circ}$, when $M A E=1.2399$. When judging the link 2 based on the $F_{\text {gof }}$ parameter, the best result was when turning the joint by $40^{\circ}$ $\left(\mathrm{F}_{\text {gof }}=71.07 \%\right)$.

\begin{tabular}{|c|c|c|}
\hline Joint angle & MAE & $\mathbf{F}_{\text {gof }}$ \\
\hline $\mathbf{2 0 ^ { \circ }}$ & 1.0928 & $72.79 \%$ \\
\hline $30^{\circ}$ & 1.0049 & $\mathbf{8 1 . 2 8 \%}$ \\
\hline $\mathbf{4 0 ^ { \circ }}$ & 1.7174 & $77.76 \%$ \\
\hline
\end{tabular}

Table 3. Overview of results of validation based on criteria MAE and Fgof

\begin{tabular}{|c|c|c|}
\hline Joint angle & MAE & $F_{\text {gof }}$ \\
\hline $20^{\circ}$ & 1.2399 & $52.20 \%$ \\
\hline $30^{\circ}$ & 1.5006 & $54.74 \%$ \\
\hline $40^{\circ}$ & 1.2571 & $71.07 \%$ \\
\hline
\end{tabular}
for link 1

Table 4. Overview of results of validation based on criteria MAE and $F_{\text {gof }}$ for link 2

\section{CONCLUSIONS}

The main goal of the research described in this paper was to validate the dynamic model of the manipulator's arm, using a simulation scheme compiled in Simulink, based on a mathematical model (listed in section 3), which also took into account the friction component. From ten measurements within one link and within the initial joint angle of $20^{\circ}, 30^{\circ}$, and $40^{\circ}$ (for each angle and the link separately), were created the arithmetic averages of these individual values from the measurements, which were used for comparison with the simulation outputs. Comparison of simulation results and averaged data was performed based on Goodness Of Fit $\left(F_{\text {gof }}\right)$ and Mean Absolute Error (MAE) indicators.

Summary of results: For the link 1, the validation output compared to the average of the measured values within the $F_{\text {gof }}$ indicator reached a value of $81.28 \%$. For the mean absolute error indicator, which represents the model error, the value of $M A E=1.0049$. These best results were obtained for the link 1 at 
a joint angle of $30^{\circ}$. The results for the link 2 were slightly worse compared to the results for the link 1 but based on the courses of the measured values and the possible oscillations present, the deterioration of the results was expected. Thus, the simulation results for the link 2 reached the value of the indicator $F_{\text {gof }}=71.07 \%$ at a joint $n$ angle of $40^{\circ}$ and $M A E=1.2399$ at an initial joint angle of $20^{\circ}$. Taking into account the presented results, the model sufficiently describes the dynamic behaviour of the system under specified conditions and therefore will be applied for further research (goal for future research - measuring a new set of data where the manipulator drive will be activated by fluid muscles and thus associated modification of the validation scheme, which was created and tested in this research, about the dynamics of the actuator.

\section{ACKNOWLEDGMENTS}

This work was supported by the projects VEGA $1 / 0393 / 18$; 055TUKE-4/2020 granted by the Ministry of Education, Science, Research and Sport of the Slovak Republic and by the Project of the Structural Funds of the EU, ITMS code: 26220220103.

\section{REFERENCES}

[Al-Qahtani 2017] Al-Qahtani, H. M. et. al. Dynamics and Control of a Robotic Arm Having Four Links. Arabian Journal for Science and Engineering, 2017, Vol. 42, No. 5, pp 1841-1852, ISSN 2191-4281

[Hosovsky 2014] Hosovsky, A. and Zidek, K. Experimental Validation of Nominal Model Characteristics for Pneumatic Muscle Actuator. Applied Mechanics and Materials: Design, Testing and Characteristics of Mechatronic Devices, Zurich, 2014 (Vol. 460). TTP, pp 1-12, ISSN 16609336

[Hosovsky 2016] Hosovsky, A. et al. Dynamic characterization and simulation of two-link soft robot arm with pneumatic muscles. Mechanism and Machine Theory, April 2016, Vol.103, pp 98-116, ISSN 0094$114 \mathrm{X}$

[Kelly 2005] Kelly, R. at el. Control of Robot Manipulators in Joint Space, Chapter 4-Case Study: The Pelican Prototype
Robot. London: Springer, 2005. ISBN 978-1-85233999-9

[Leborne 2018] Leborne, F. et al. Dynamic Modeling and Identification of an Heterogeneously Actuated Underwater Manipulator Arm. In: 2018 IEEE International Conference on Robotics and Automation (ICRA), Brisbane, Australia, 21-25 May, 2018. Danvers: IEEE, pp 4955-4960, ISSN 2577-087X

[Pal 2017] Pal, R. Predictive Modeling of Drug Sensitivity, Chapter 4 - Validation methodologies. Massachusetts: Academic Press, 2017. ISBN 978-012-805274-7

[Papageorgiou 2020] Papageorgiou, D. et al. Online friction parameter estimation for machine tools. Advanced Control for Applications, 2020, Vol.2, No.1, pp 1-27, ISSN 2578-0727

[Pitel 2014] Pitel, J. and Tothova, M. Dynamics of pneumatic muscle actuator: Measurement and modeling. In: Proceedings of the 2014 15th International Carpathian Control Conference (ICCC), Velke Karlovice, 28-30 May, 2014. Danvers: IEEE, pp 432436. ISBN 978-1-4799-3527-7

[Siciliano 2009] Siciliano, B. et. al. Robotics: Modelling, Planning and Control. London: Springer-Verlag, 2009, ISBN 978-1-84628-641-4

[Spong 2020] Spong M. W. et. al. Robot Modeling and Control. John Wiley \& Sons, 2020, pp 608, ISBN 978-1-11952399-4

[Swevers 2007] Swevers, J. et. al. Dynamic Model Identification for Industrial Robots. IEEE Control Systems Magazine, 2007, Vol. 27, No. 5, pp 58-71, ISSN 1941000X

[Trojanova 2018] Trojanova, M and Hosovsky, A. Comparison of Different Neural Networks Models for Identification of Manipulator Arm Driven by Fluidic Muscles. Acta Polytechnica Hungarica, 2018, Vol. 15, No. 7, pp 728, ISSN 1785-8860

[Trojanova 2019] Trojanova, $M$ and Hosovsky, A. NARX-NN Model of 2-DOF Robot Arm Dynamics Driven by Fluidic Muscles. MM Science Journal, 2019, December 2019, pp 3458-3464, ISSN 1803-1269

\section{CONTACTS:}

Ing. Monika Trojanova, PhD., Ing. Tomas Cakurda

Technical University of Kosice, Faculty of Manufacturing Technologies with the Seat in Presov, Department of Industrial Engineering and Informatics, Bayerova 1, 08001 Presov, Slovakia +421 055602 6420, monika.trojanova@tuke.sk, tomas.cakurda@tuke.sk, http://www.fvt.tuke.sk 